\section{Review Article}

Clinical Chemistry

Ann Lab Med 2020;40:285-296

https://doi.org/10.3343/alm.2020.40.4.285

Check tor
updates

ISSN 2234-3806 elSSN 2234-3814
ANNALS OF

LABORATORY

MEDICINE

\title{
Cortisol Measurements in Cushing's Syndrome: Immunoassay or Mass Spectrometry?
}

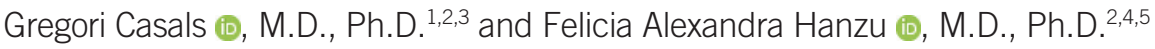 \\ ${ }^{1}$ Department of Biochemistry and Molecular Genetics, Hospital Clínic, Barcelona, Spain; ${ }^{2}$ Institut d'Investigacions Biomèdiques August Pi i Sunyer \\ (IDIBAPS), Barcelona, Spain; ${ }^{3}$ Centrode Investigación Biomédica en Red de Enfermedades Hepáticas y Digestivas (CIBERehd), ISCIII, Madrid, Spain; \\ ${ }^{4}$ Department of Endocrinology and Nutrition, Hospital Clínic, Barcelona, Spain; ${ }^{5}$ Department of Medicine, Faculty of Medicine and Health Sciences, \\ University of Barcelona, Barcelona, Spain
}

Determination of cortisol levels in the urine (24 hours urine free cortisol), saliva (late-night), or serum (total cortisol after dexamethasone suppression) is recommended to screen for Cushing's syndrome (CS). This review focuses on the differences between the frequently used cortisol-antibody immunoassay-based methods and the highly specific mass-spectrometry-based methods that are progressively being employed in clinical laboratories for CS screening. The particular characteristics of cortisol metabolism and the lack of specificity of the immunoassays cause marked differences between both methods that are in turn highly dependent on the biological matrix, in which the cortisol is measured. Understanding the origin of these differences is essential for the interpretation of these results. Although cross-reactivity with endogenous steroids leads to grossly inaccurate results of immunoassay measurements of cortisol in the saliva and urine, preliminary evidence suggests that the clinical sensitivity of CS screening using immunoassays may be similar to CS screening using mass spectrometry. However, mass spectrometry offers more accurate results and considerably reduced variation across laboratories, while avoiding false-positive results. Moreover, mass spectrometry can overcome some common diagnostic challenges, such as identification of exogenous corticosteroids or simultaneous assessment of appropriate dexamethasone levels in suppression tests. Further, comprehensive mass spectrometry-based profiling of several steroid metabolites may be useful for discriminating among different subtypes of CS. Finally, this review discusses the main preanalytical factors that could cause variations in cortisol measurements and their influence on the reliability of the results.

Key Words: Cortisol, Immunoassay, Mass spectrometry, Sensitivity
Received: November 8, 2019

Revision received: December 20, 2019

Accepted: February 7, 2020

\section{Corresponding author:}

Gregori Casals, M.D., Ph.D.

Department of Biochemistry and Molecular

Genetics, Hospital Clínic Universitari,

Villarroel 170, Barcelona 08036, Spain

Tel: + (3493)2275400 × 2667

Fax: +(3493)2275697

E-mail: casals@clinic.cat

\section{INTRODUCTION}

Cushing's syndrome (CS) is the state of hypercortisolism that results from chronic exposure to excessive circulating levels of endogenous or exogenous glucocorticoids. Endogenous hypercortisolism is usually related to excessive adrenocorticotropic hormone (ACTH) production due to the presence of pituitary adenomas (Cushing's disease) or the autonomous production of cortisol by the adrenal glands (adrenal Cushing's). In some cases,
CS is caused by the ectopic production of ACTH or corticotrophin-releasing hormone $(\mathrm{CRH})$ from extrapituitary tumors. In addition to endogenous hypercortisolism, an excess of any glucocorticoid administered via different routes can also give rise to exogenous CS. CS is a potentially lethal disorder that is associated with various comorbidities that increase morbidity and mortality, including cardiovascular disease, diabetes, hypertension, a hypercoagulable state, fractures, or infections [1-3].

The clinical features of CS may show a wide spectrum of ab- 
normalities from mild to severe manifestations. The classical signs of the disease in its most obvious form are less frequently observed in current clinical practice and have a tendency toward being milder. Moreover, the clinical diagnosis of CS may be equivocal because many of the typical symptoms, such as weight gain and hypertension, are also very common in the general population. In particular, mild CS may show a nearly identical clinical profile to that of the highly prevalent metabolic syndrome [4].

Accordingly, clinical diagnosis of CS is challenging, particularly in a population with a high prevalence of metabolic syndrome, and several different assays are often required to make a correct diagnosis. Mildly abnormal results, together with the typical phenotypic characteristics of CS, may occur in a variety of conditions that physiologically activate the hypothalamic-pituitary-adrenal axis, such as alcoholism, depression, or obesity, among others (i.e., pseudo-CS) [5, 6], and pregnancy [7]. Moreover, subclinical CS can present as mild hypercortisolism without the classical clinical manifestations [8]. Finally, some patients may present with episodic secretions of cortisol along with fluctuating symptoms and signs, due to a state of cyclical CS [9].

In this regard, there is increasing evidence of the value of hair cortisol level as a marker of long-term, systemic exposure to cortisol in patients with CS, which is particularly helpful to identify patients with milder forms or cyclical CS [10-12]. Further advantages of this method include easy sample collection, storage and transport at room temperature, and ability to provide retrospective information about systemic cortisol exposure over months, which can potentially facilitate earlier diagnosis and treatment. Moreover, the sensitivity and specificity of hair cortisol measurement for CS diagnosis are both high [11], and there is a direct correlation between monthly cumulative cortisol exposure per centimeter of hair segment in both healthy subjects and patients with CS [12].

Upon clinical suspicion, the basis for establishing CS diagnosis is the biochemical confirmation of autonomous hypercortisolism. Biochemical assays to confirm CS diagnosis are designed according to the main physiopathological characteristics of CS, including the loss of both the normal circadian rhythm and feedback of the hypothalamic-pituitary-adrenal axis, which result in chronic exposure to excessive circulating levels of cortisol [13]. In this regard, the 2008 Endocrine Society clinical practice guidelines recommend measurement of the levels of late-night salivary cortisol (LNSC), 24-hour urinary free cortisol (UFC), and serum cortisol after dexamethasone-suppression test (DST) as screening tests for CS [14].
CS is a rare disease with similar clinical features to other conditions, such as metabolic syndrome or osteoporosis. To increase the likelihood of CS detection in populations susceptible to the abovementioned diseases, screening tests for CS need to be highly sensitive. Therefore, following a positive screening test, further biochemical characterization is necessary. Based on etiology, endogenous CS can be broadly classified into two categories: ACTH-dependent and ACTH-independent CS. Thus, a biochemical assay for plasma ACTH is important for further localization studies and appropriate management of the disease. In addition, the $\mathrm{CRH}$ stimulation test, desmopressin (dDAVP) stimulation test, high-dose DST, and bilateral inferior petrosal sinus sampling may be useful in specific situations [15, 16]. Moreover, CS has a high likelihood of recurrence [17], which may require a long-term biochemical follow-up of the patients after treatment.

Given this scenario, biochemical determination plays a central and increasingly important role in the management of CS. Currently, there are reference measurement procedures for serum cortisol but not for urine or salivary cortisol. Nevertheless, the main routine cortisol assays are significantly biased, and there is currently no implementation of metrologically traceable results in clinical practice, resulting in poor standardization of routine assays. Therefore, structure-based assays, such as mass spectrometry, are recommended [14]. This review focuses on the main methods for screening CS by measuring cortisol in the serum, saliva, and urine and the influence of the main biochemical characteristics of the analytes and preanalytical aspects on the different analytical outcomes. We particularly focus on the differences between the more frequently used cortisol-antibody immunoassay-based methods and the highly specific mass spectrometry determinations that are progressively being incorporated in clinical laboratories.

\section{URINE CORTISOL}

\section{Physiology}

Cortisol exists in the urine in free (unconjugated) and conjugated (e.g., glucuronide-conjugated and sulfate-conjugated) forms. Approximately $1-2 \%$ of protein-unbound circulating cortisol is excreted in the urine; therefore, the level of cortisol in the urine reflects the level of the protein-unbound (biologically active or free) form of cortisol in the plasma. Determination of the UFC is a well-established screening tool for CS [14]. Owing to the circadian rhythm of cortisol excretion, this assay is performed with a 24-hour collection of urine as an equivalent of the time-integrated 
tissue exposure to free cortisol over a day. UFC measurements are not affected by diurnal variation or changes in cortisol-binding proteins in the serum.

\section{Sampling and preanalytics}

Patients are required to collect their urine over a 24-hour period after discarding the first morning void. All subsequent voids during the day, night, and the first morning void of the following day should be included in the collection. It is recommended that the urine is kept refrigerated during the collection period [14, 18] and that the sample is immediately frozen once received at the laboratory. However, the UFC was reported to be stable for over three days irrespective of the storage temperature $\left(4^{\circ} \mathrm{C}\right.$ vs room temperature) or exposure to light (darkness vs brightness) [19]. Unpreserved urine samples can also be used for the measurement of UFC since urine $\mathrm{pH}$ also has no substantial effect on the measurements [20].

The biological within-subject variation of UFC is high (>60\%) and is even higher than the between-subject variation [21]. The upper limit of the reference range of the assay is usually used as the criterion for a positive result, and at least two measurements of UFC are recommended for CS screening [14]. Since complete collection of 24-hour urine samples may be cumbersome, written instructions for the patients are suggested to avoid inaccurate collection. Thus, false-negative cortisol results may occur because of incomplete urine collection, whereas urine collection over 24 hours can result in falsely elevated UFC levels. In this regard, measurement of urine creatinine can help assess the completeness of the 24-hour collection or make the decision to discard any overcollection [22]. Given that creatinine excretion tends to remain constant if the glomerular filtration rate is normal, the normalized urine cortisol:creatinine ratio may also be useful. However, UFC is not recommended for CS screening in patients with moderate-to-severe renal impairment, as significantly lower UFC values are observed when the creatinine clearance falls below $60 \mathrm{~mL} / \mathrm{min}$ [23]. For reliable UFC measurements, it is also important to avoid drinking excessive amounts of fluid or using any glucocorticoid during the collection period.

\section{Immunoassay measurements}

Immunoassay is the most routine and common method for the determination of UFC in many clinical laboratories. In addition to unconjugated cortisol (i.e., UFC), the urine also contains cortisol in conjugated form, along with other highly abundant conjugated and unconjugated cortisol metabolites with chemical structures similar to that of cortisol. These metabolites may cross- react with the antibodies of the immunoassay and thus interfere with UFC measurements. Therefore, it is essential to perform an extraction step prior to the immunoassay [24], which typically involves mixing the urine with an organic solvent (e.g., dichloromethane or ethyl acetate) and removing the aqueous layer; since cortisol is less water-soluble than most of its metabolites, it can be easily separated in this process. Solid-phase extraction using disposable cartridges may also be used for extraction. Since this pre-analysis extraction step is time-consuming and requires technical expertise, some immunoassay manufacturers do not explicitly require this, despite its recognition as crucial before immunological measurements [24]. Currently, extraction is usually performed only in tertiary-care centers or academic hospitals.

Despite effectively removing several interfering molecules, the extraction step cannot completely avoid the cross-reactivity problem since some interfering components remain. In this regard, it is important to consider that determination of UFC by an immunoassay is mainly based on serum cortisol and is particularly vulnerable to interference. Thus, the most commonly used antibody in cortisol immunoassays is raised against cortisol-3-(ocarboxymethyl)-oxime conjugates [25], which provides acceptable specificity for serum assays. However, levels of ring-A cortisol metabolites, such as dihydrocortisol, tetrahydrocortisol, and other related substances that may be abundant in the extracted urine, introduce quantitatively significant positive bias.

\section{Mass spectrometry measurements}

Mass spectrometry is a powerful structural characterization technique that overcomes the antibody-antigen cross-reactivity problem of immunoassays. At present, mass spectrometry is considered the most specific method to determine the true UFC [14]. Comparison of UFC results obtained by immunoassay with those obtained by mass spectrometry showed that results obtained by immunoassay were overestimated by approximately two folds [25-27]; however, this overestimation is variable and depends on differences in the degrees of cross-reactivity of the various cross-reacting metabolites in each immunoassay. As a result, important differences in reference ranges are observed, depending on whether the UFC is measured by an immunoassay or mass spectrometry. On the basis of its high analytical specificity, mass spectrometry measurements of UFC are increasingly recommended, demonstrating high diagnostic accuracy for CS [28, 29].

Analytically, the goal should be to measure cortisol level as accurately and precisely as possible. In this regard, mass spectrometry is considered to be the best analytical method to mea- 


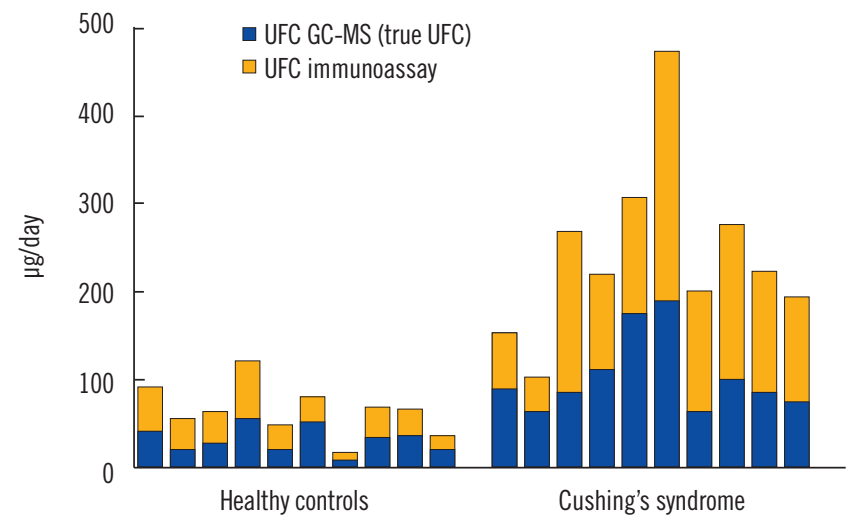

Fig. 1. Representation of the bias introduced by immunoassays in UFC measurements in 10 healthy control subjects and 10 patients with CS (unpublished data). UFC was simultaneously measured by an immunoassay, as described previously (Liaison, Diasorin, Italy), and GC-MS. The mean positive biases introduced by the immunoassay in all healthy control subjects and CS patients were determined to be $121 \%$ (range: $44-219 \% ; N=33$ ) and $135 \%$ (range: $33-344 \%$; $\mathrm{N}=35$ ), respectively.

Abbreviations: UFC, urinary free cortisol; CS, Cushing's syndrome; GC-MS, gas chromatography-mass spectrometry.

sure the true cortisol level. In fact, awareness of the important lack of specificity of immunoassays for UFC measurements begs to question what each immunoassay is really measuring, and even calls into question whether the term "UFC" should be applied to such an inaccurate methodology [30]. Fig. 1 provides an example of the gross bias introduced by immunoassays used for UFC measurements. Other key limitations of UFC immunoassays include the absence of recovery correction, interference with exogenous steroids, and variability among manufacturers.

\section{Diagnostic clinical value}

Despite the above-mentioned limitations of immunoassays, from the perspective of the diagnostic value for the screening of CS, the issue of cross-reactivity with cortisol metabolites may actually be an advantage for improving the detection of overall glucocorticoid production, resulting in enhanced diagnostic sensitivity compared with the high specificity for UFC measurement offered by mass spectrometry [31]. Two studies that directly compared the diagnostic performance for CS between mass spectrometry and immunoassay did not find significant differences [26, 32]. Moreover, the impact of the differences between the two methods in specific situations remains unclear, such as in the case of advanced liver disease or in the medical treatment of CS, since changes in hepatic metabolism have minimal effect on urinary cortisol but greater effects on urinary cortisol metabolites [33].

\section{Synthetic steroids}

In addition to cross-reactivity with cortisol metabolites, cross-reactivity in immunoassays can also occur with synthetic steroids that are structurally related to cortisol. Although it is recommended that clinicians ensure that patients are not on exogenous glucocorticoid therapy at the time of urine collection for UFC measurements, this is not always possible, and the patients may also be unaware that they are receiving such therapy. Although many exogenous corticosteroids may be detected in varying degrees across different cortisol immunoassays, manufacturers typically only provide information on the potential cross-reactivity for some of these corticosteroids. Consequently, it is often unknown if a specific corticosteroid will have a cross-reaction and to what extent with a particular immunoassay. Compounding this problem, exogenous steroids are associated with endogenous suppression of the adrenal axis, which imposes a further challenge in the interpretation of results [34, 35]. In this scenario, mass spectrometry may be extremely useful for the differentiation of endogenous hypocortisolism and adrenal axis suppression due to the presence of synthetic corticoids and for the detection of factitious CS [36]. Djedovic, et al. [37] found that samples from eight of 154 (5.2\%) patients screened for suspected CS showed detectable concentrations of synthetic glucocorticoids: four were positive for dexamethasone, one was positive for betamethasone, and three were positive for prednisolone. Dexamethasone and betamethasone do not generally cross-react in immunoassays, whereas prednisone/prednisolone shows cross-reactivity. Interestingly, chromatographic methods coupled to mass spectrometry allow for the simultaneous detection and identification of many synthetic compounds in a single analysis $[37,38]$. Despite their high specificity, interference remains a possibility with mass spectrometry. Thus, in the case of cortisol measurements, prednisolone interference should be considered, owing to its structural similarity with cortisol.

\section{Endogenous steroids}

In addition to exogenous steroids, mass spectrometry can also measure specific endogenous cortisol metabolites [39-41]. However, further studies are needed to define whether these metabolites provide additional information beyond the measurement of UFC in certain diseases related to CS, such as adrenal incidentalomas [42]. Recently, an increased urinary cortisol:cortisone ratio was proposed to be a useful marker for differentiating CS and ectopic ACTH syndrome [43]. Normal levels of free cortisone are similar to those of UFC, which may also be measured by an immunoassay or mass spectrometry. 


\section{Method validation}

Despite its great potential for giving highly specific measurements, mass spectrometry methods should also be validated [44], and their reference range should also be determined. Recently, Brossaud, et al. [45] demonstrated good agreement of UFC results obtained using four different in-house mass spectrometry assays for 98 urine samples ( 20 from CS patients and 78 from control subjects). However, for extreme UFC values, a negative or positive bias was noted, showing that variation in the results can occur for mass spectrometry depending on the analytical process. The normal UFC ranges obtained from the same samples were determined to be 17-126, 15-134, 12-118, and 27-157 nmol/day [45].

\section{SALIVARY CORTISOL}

\section{Physiology}

Salivary cortisol reflects the changes in free (unbound) serum cortisol and, similar to UFC, is considered a reliable alternative to measuring free cortisol in the serum. According to the free hormone hypothesis, the level of an unbound form of a hormone determines its biological activity. The physiological basis for the measurement of LNSC is that the loss of circadian rhythm, with the absence of a late-night cortisol nadir, is a consistent biochemical characteristic in patients with CS [14]. Since salivary cortisol adequately reflects the circadian rhythm of circulating cortisol, LNSC is recommended as a first-line screening test for CS $[14,46,47]$.

\section{Sampling and preanalytics}

To measure LNSC, late-night saliva samples are collected between 23:00 hours and 0:00 hours, usually using a cylindrical absorbent cotton swab (salivette). However, a recent study showed lower levels of salivary cortisol when healthy adult subjects conducted their sampling at their normal bedtime than when they were forced to remain awake until 23:00 hours or later [48]. Saliva production is similar among subjects and, compared with 24-hour urine, the effect of sample volume on LNSC measurements is much lower. In addition, salivary measurements have the advantage of being noninvasive, which also favors the acceptance of serial determinations.

However, greater practicability of LNSC determination (compared with that of midnight cortisol serum determination, for example) has a disadvantage of the loss of control in sample collection by the laboratory. Therefore, special care should be taken to explain the procedure and timing of sample collection to the patient. It is generally recommended to collect saliva at least 30 minutes after eating, drinking, or tooth brushing. Falsely elevated salivary cortisol levels may be related to sample contamination with blood, licorice, or smoking before sample collection [49].

Given that the hypercortisolism of CS can be variable, at least two measurements of salivary cortisol are recommended [14]. The biological within-subject variation of LNSC in healthy subjects was reported to be 34\% [50]; however, extreme fluctuations of LNSC levels are common in patients with Cushing's disease [51]. Advanced age is also associated with higher levels of LNSC and has no evident influence of gender or body mass index [52].

Cortisol in saliva samples tends to be stable, and patients can store their samples at $4-8^{\circ} \mathrm{C}$ in their home refrigerator for up to seven days before delivery to the laboratory. Once received at the laboratory, the samples may be centrifuged to obtain the supernatant, which can then be frozen at $-20^{\circ} \mathrm{C}$ until analysis. Salivary cortisol samples were found to be stable under storage at $5^{\circ} \mathrm{C}$ for up to three months and under storage at $-20^{\circ} \mathrm{C}$ for up to 12 months, whereas the cortisol levels decreased by $9.2 \%$ per month of storage at room temperature [53].

\section{Variability across laboratories}

Significant differences in LNSC results are found across different laboratories [53]. Some of the possible explanations for these differences include variation in saliva sample collection devices or protocols, sample handling and transport, number and frequency of sampling, compliance with collection timing or sample contamination, and variation in the performance of assays across laboratories. Calvi, et al. [54] evaluated the sources of measurement variability in the assay of salivary cortisol within and between established international academic-based laboratories that are specialized in saliva analyses. They found that $90 \%$ of the variance in salivary cortisol levels was attributable to differences related to the sample itself, $8 \%$ was attributable to differences between laboratories, and $2 \%$ was attributable to differences between duplicate assays of the same sample within laboratories [54]. Given that differences in the recovery between collection devices may be high, the same device should be used or the reference intervals should be reassessed if the device is modified. The absence of certified reference materials, and the lack of harmonization or deficit of external proficiency-testing programs may explain the persistence in the variance of salivary cortisol levels between methods. 


\section{Immunoassay measurements}

Similar to UFC, immunoassays and mass spectrometry are the main methods used to measure salivary cortisol. The advantages of immunoassays include easy performance and small volume requirements of saliva. In addition to specific immunoassays for salivary measurements, automated serum cortisol immunoassays have been adapted to measure the level of cortisol in saliva [52, 55-57]. In this case, it is important to confirm that the limit of quantification of the assay is adequate to measure the low salivary cortisol levels found at the nadir of the diurnal rhythm.

Similar to the case of UFC measurements, the analytical specificity of saliva immunoassays may be limited by antibody crossreactivity with other steroids present in the sample. In the case of saliva, significantly higher antibody cross-reactivity may be present with cortisone, which is present at high levels in saliva. In fact, after free cortisol enters the saliva by diffusion, it is rapidly converted to cortisone by the enzyme 11 beta-hydroxysteroid dehydrogenase type 2 , resulting in four to nine-times greater cortisone levels than cortisol levels in saliva [58]. In addition to cortisone, other steroids may also present cross-reactivity with immunoassays of saliva cortisol, posing a challenge for accurately measuring the low levels of cortisol in late-night saliva samples. In addition, synthetic steroids, such as prednisone/prednisolone, may also cross-react in some immunoassays and produce elevated results, particularly when the saliva is obtained just after administration of an oral dose. Different cross-reactivities can influence the cut-off values and clinical performance of LNSC assays [59].

\section{Mass spectrometry measurements}

Mass spectrometry measurements of salivary cortisol eliminate the influence of the cross-reactivity with cortisone and other steroids, thereby offering a more specific analytical method. Consequently, liquid chromatography-tandem mass spectrometry (LC-MS/MS) results may be substantially lower than radioimmunoassay results [60], enzyme immunoassay [48], or automated chemiluminescence immunoassay [56]. Table 1 shows the ref-

Table 1. Mass spectrometry and immunoassay late-night salivary cortisol (LNSC) reference values in healthy volunteers

\begin{tabular}{llcccc}
\hline Method & \multicolumn{1}{c}{ Assay } & $n$ nmol/L & N & Year & Reference \\
\hline RIA & Coat-a count & $<2.9$ & 73 & 1998 & {$[76]$} \\
RIA & SmithKline Bioscience & $<13.6$ & 34 & 2002 & {$[77]$} \\
RIA & Byk-Sangtec Diagnostica & 10.4 & 27 & 2003 & {$[78]$} \\
RIA & CIS Biointernational & 5.5 & 54 & 2004 & {$[79]$} \\
RIA & Cis Biointernational & $0.3-4.3$ & 20 & 2005 & {$[80]$} \\
RIA & Esoterix & $1.4-4.7$ & 26 & 2007 & {$[49]$} \\
EIA & Salimetrics & $<4.3$ & 20 & 2011 & {$[50]$} \\
EIA & BUSCAR & $<3.2$ & 30 & 2015 & {$[81]$} \\
ELISA & IBL International & $0.83-8.3$ & 725 & 2016 & {$[82]$} \\
EIA & Salimetrics & $<2.3^{*}$ & 54 & 2019 & {$[48]$} \\
ECLIA & Elecsys 2010, Roche & $0.2-8.9$ & 100 & 2006 & {$[56]$} \\
ECLIA & Elecsys 2010, Roche & $1.9-9.0$ & 42 & 2015 & {$[83]$} \\
ECLIA & Cobas 601, Roche & $<8.3$ & 122 & 2017 & {$[52]$} \\
ECLIA & Elecsys 2010, Roche & $0.11-3.42$ & 57 & 2018 & {$[84]$} \\
ECLIA & Access, Beckman Coulter & $<15.3$ & 117 & 2019 & {$[57]$} \\
Mass spectrometry & LC-MS & $<2.8$ & 102 & 2012 & {$[85]$} \\
Mass spectrometry & LC-MS & $<2.8$ & 60 & 2013 & {$[86]$} \\
Mass spectrometry & LC-MS & $0.25-2.5$ & 91 & 2015 & {$[87]$} \\
Mass spectrometry & LC-MS & $<3.1$ & 52 & 2018 & {$[61]$} \\
Mass spectrometry & LC-MS & $<1.5^{*}$ & 54 & 2019 & {$[48]$} \\
\hline
\end{tabular}

*Patient's usual bedtime (20:25-24:00 hours).

Abbreviations: RIA, radioimmunoassay; EIA, enzyme immunoassay; ELISA, enzyme-linked immunosorbent assay; ECLIA, electrochemiluminescence immunoassay; LC-MS, liquid chromatography-mass spectrometry. 
erence values obtained in healthy subjects across different studies using different methods. In contrast to the abundant data available for the diagnostic value immunoassays, the diagnostic value of LNSC determined by mass spectrometry in clinical practice has only been evaluated in a few studies, and there are few direct comparisons between the two methods. Therefore, further work is needed to establish whether the more analytically specific mass spectrometric measurements provide greater diagnostic accuracy for CS or whether the cross-reactivity of immunoassays may paradoxically result in better diagnostic sensitivity [5, 31, 60]. Recently, Mészáros, et al. [61] found similar diagnostic values for CS between an LC-MS/MS method and electrochemiluminescent immunoassay, although the levels obtained using the latter method were around 30\% higher. In addition, Ceccato, et al. [57] used mass spectrometry to evaluate selected unexpected results obtained with a chemiluminescence immunoassay and suggested that confirmation of immunoassay results by mass spectrometry may improve the overall diagnostic accuracy.

Mass spectrometric measurements may be also useful to detect specific synthetic steroids. Simultaneous measurements of natural and synthetic glucocorticoids may enable the rapid identification of surreptitious medication or contamination of saliva [62]. Although the mass spectrometry measurements used in routine clinical practice cannot distinguish between exogenous and endogenous cortisol, simultaneous measurement of salivary cortisol and cortisone may help identify samples contaminated with topical hydrocortisone based on an elevated cortisol:cortisone ratio [63].

Despite its high specific potential, mass spectrometry methods for LNSC may not be completely free of interference with other exogenous steroids, such as prednisolone or endogenous metabolites. Indeed, Israelsson, et al. [64] recently reported that the isomers 20 $\alpha$ - and 20ß-dihydrocortisone had similar chemical properties and fragmentation patterns as cortisol when detected in positive-ion mode using electrospray ionization (ESI). Therefore, validation of LC-MS/MS-ESI methods for cortisol de-

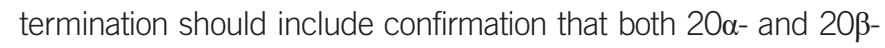
dihydrocortisone are separated from cortisol at the chromatography step.

\section{SERUM OR PLASMA CORTISOL}

\section{Physiology}

Approximately $80 \%$ of circulating cortisol is bound to cortisolbinding-globulin (CBG), $10 \%$ is bound to albumin, and $10 \%$ is in an unbound (free) form [33]. Although the measurement of free plasma cortisol is of the greatest clinical interest, it is laborintensive, time-consuming, and methodologically difficult to implement in routine clinical practice owing to the need for a preanalysis step to separate the bound and unbound fractions of cortisol by equilibrium dialysis, gel filtration, or ultrafiltration. As an alternative, measurement of the total (bound and unbound) cortisol with an immunoassay is a fast and reproducible approach and is therefore the current method of choice in many clinical chemistry laboratories. However, the measurement of serum or plasma total cortisol may be affected by changes in CBG and/or albumin levels [33] and may not reflect changes in the free hormone levels.

\section{Sampling and preanalytics}

Together with UFC and LNSC measurements, overnight DST is recommended for the initial screening of CS [14]. In addition, the overnight DST is currently recommended as the first assay to detect cortisol excess in patients with an adrenal incidentaloma [65]. In patients with endogenous CS, the normal suppression of ACTH and cortisol fails when low doses of dexamethasone are administered [14]. The overnight DST can be easily performed as an outpatient assay. Various doses of dexamethasone have been used for this purpose, but $1 \mathrm{mg}$ dexamethasone is commonly provided to the patient in an oral form between 23:00 hours and 0:00 hours, after which the cortisol level is measured between 8:00 hours and 9:00 hours the next morning. A total cortisol plasma level below $50 \mathrm{nmol} / \mathrm{L}(1.8 \mu \mathrm{g} / \mathrm{dL})$ is considered as an appropriate response to the test $[1,14,66]$, although there is some controversy and different serum cortisol cut-off values have been proposed ranging from 50 to $138 \mathrm{nmol} / \mathrm{L}$ (1.8-5 $\mu \mathrm{g} / \mathrm{dL})$ [18]. A negative DST, using a cut-off value of 50 $\mathrm{nmol} / \mathrm{L}$ virtually excludes the possibility of autonomous cortisol secretion in the adrenal incidentalomes, whereas a value above $138 \mathrm{nmol} / \mathrm{L}$ is highly suggestive of autonomous cortisol secretion. In cases of cortisol values between 50 and $138 \mathrm{nmol} / \mathrm{L}$ the term "possible autonomous cortisol secretion" has been proposed [65].

\section{Factors affecting cortisol serum levels}

A variety of clinical and analytical conditions can affect the serum cortisol results after the DST. First, drugs that induce or inhibit hepatic CYP3A4 may accelerate or inhibit dexamethasone metabolism and cause false-positive or false-negative results by decreasing or increasing serum cortisol levels, respectively. In addition, dexamethasone clearance may be reduced in patients 
with liver and/or renal failure. In addition, the dependence of total cortisol measurements on transport proteins may also influence the results. For example, estrogens increase circulating CBG levels and are associated with high false-positive rates $(\sim 50 \%)$ in the overnight DST for women taking the oral contraceptive pill or under hormone replacement therapy [14]. Therefore, estrogen-containing medications are recommended to be withdrawn for six weeks before the test [14]. Recently, Vastbinder, et al. [67] found that eight of 13 (62\%) female volunteers who were using oral contraceptives showed inadequate suppression of cortisol upon being administered 1 mg dexamethasone. One week after the contraceptive was withdrawn, the number of false-positive results decreased to 1 (8\%), and all tests were normal after six weeks, suggesting that the recommended six-week period may be shortened. Conversely, the reduction of transport proteins found in patients with critical illness or advanced-stage renal disease may result in decreased serum cortisol levels [14]. In addition to variations in metabolism, the variable absorption of dexamethasone may also explain the interindividual variation in assay results, even for healthy subjects on no medication.

\section{Simultaneous dexamethasone measurements}

Considering the several sources of variation, it may help measure both cortisol and dexamethasone to ensure adequate plasma dexamethasone levels, which may be useful to evaluate false positive and false negative responses [68]. Hempen, et al. [69] used an LC-MS/MS approach to simultaneously measure serum cortisol and dexamethasone in 62 post-DST samples of 46 patients with suspected CS and 16 healthy control subjects. The range of dexamethasone levels was 3.3-19.6 nmol/L, similar to that previously observed by radioimmunoassay [68]. Interestingly, five patients (11\%), whose cortisol values would have been interpreted as not suppressible, presented dexamethasone levels below the limit of quantification, suggesting lack of dexamethasone compliance or alterations in dexamethasone absorption or metabolism. Conversely, three patients (6.5\%) presented non-suppressed levels of cortisol accompanied by a sufficient dexamethasone level, which further improved the interpretation of the post-DST result [69]. More recently, Ueland, et al. [70] prospectively evaluated the simultaneous measurements of serum cortisol and dexamethasone by LC-MS/MS in the DST for 302 subjects. They found that dexamethasone measurements reduced false-positive DST results by $20 \%$ and improved the diagnostic specificity. Interestingly, the same study showed that post-DST salivary cortisol and cortisone levels were strongly correlated with post-DST serum cortisol levels. In fact, post-DST salivary cortisone was a better discriminatory marker between healthy subjects and patients with CS or with autonomous cortisol secretion than salivary cortisol.

\section{Immunoassay and mass spectrometry measurements}

Similar to UFC and LNSC measurements, plasma cortisol measurements may be affected by the type of assay used. More than 30 different immunoassays are currently available as manual kits and/or automated platforms [5]. Direct automated nonisotopically labeled immunoassays are usually used in routine clinical practice. However, agreement between immunoassays is variable, and although the results may be comparable to those of mass spectrometry, according to some studies [71, 72], other studies showed slightly higher levels obtained using the immunoassay than using mass spectrometry. El-Farhan, et al. [73] found that the immunoassay bias ranged from 1.08 to 1.36 when comparing gas chromatography-mass spectrometry measurements to those obtained with the automated immunoassays Advia Centaur (Siemens), Architect (Abbott), Modular Analytics E170 (Roche), Immulite 2000 (Siemens), and Access (Beckman). Thus, direct serum cortisol immunoassays may also present analytical specificity-related difficulties, although to a lesser extent than urine-based assays. In direct immunoassays, the serum is analyzed without a previous extraction step, which may favor matrix interference and cross-reactivity with other structurally similar steroids. The specific cross-reacting steroids and degree of reactivity depend on the specific immunoassay used, although prednisolone and 11-deoxycortisol are common interferents. In particular, the absence of reactivity with dexamethasone in the cortisol immunoassay should be confirmed when performing cortisol measurements after the DST. Moreover, chemical reactions in direct assays may be affected by plasma proteins, as endogenous plasma proteins compete with the antibody against cortisol in the sample. Although a blocking agent is usually added to suppress this effect, it may not be completely eliminated [24].

Differences between serum cortisol immunoassays may ultimately affect the interpretation of the results of the DST and put into question the need for method-specific cut-off values over the applicability of a single universal cut-off value. Brixey-McCann, et al. [71] compared the serum cortisol measurement results obtained by mass spectrometry and the automated immunoassays Architect (Abbott), E170 (Roche), Access (Beckman), and Centaur (Siemens). The interpretation of the DST results was discrepant when using a cut-off value of $50 \mathrm{nmol} / \mathrm{L}$ in 19 of $108(17.6 \%)$ samples, with clinical sensitivities ranging from 
$87 \%$ to $94 \%$ depending on the assay.

Differences among immunoassays and between immunoassays and mass spectrometry may be greater in patients with CS that are under active medical treatment. Monaghan, et al. [72] found that the bias between LC-MS/MS assays and the Siemens ADVIA Centaur XP immunoassay was between $12.6 \%$ and $90.8 \%$ in patients receiving metyrapone. This high bias may be attributed to the cross-reactivity with 11-deoxycortisol and other structural analogues preceding cortisol in the steroid biosynthesis pathway, which are found at elevated levels in these patients due to the steroidogenic inhibitory effect of the drug. As a result, immunoassays may not adequately reflect the true cortisol values in these patients, which could lead to unrecognized hypoadrenalism, in turn presenting symptoms that can be confused with the side effects of metyrapone.

Finally, the advantage of mass spectrometry to simultaneously analyze many plasma steroids has been considered a breakthrough, and several authors have suggested mass spectrometry as the most promising tool for the diagnosis of subclinical CS [74] and the identification of different subtypes of CS [75], suggesting the potential of a single mass spectrometric assay to achieve an etiological diagnosis.

\section{CONCLUSIONS}

Preanalytical factors greatly influence the results of cortisol measurements for all sample types (UFC, LNSC, or serum after the DST). Therefore, identifying and properly controlling these fac- tors is essential for the correct assessment and diagnosis of CS. Cortisol determination for the evaluation of CS in clinical laboratories has traditionally been performed by immunoassays. Currently, mass spectrometry measurements are also being employed for CS evaluation. Different analytical and clinical possibilities of mass spectrometry measurements for CS patients are summarized in Table 2.

The problems of specificity due to cross-reaction of cortisol immunoassays with endogenous steroids results in high inaccuracy and variability across laboratories. Mass spectrometry allows overcoming these limitations and gives results that are more accurate and similar. However, recent reports suggest that, mass spectrometry may present a clinical sensitivity for CS screening similar to that of many immunoassays already in use. In contrast, mass spectrometry may present high clinical specificity and could be used to reduce the number of false-positive results obtained by immunoassay. In addition, as CS is difficult to diagnose, mass spectrometry has been shown to reduce the uncertainty in clinical results, which is a problem posed by immunoassays. Mass spectrometry allows for the identification of exogenous corticosteroids, which are the most common cause of CS, and thus allows the confirmation of a clinical suspicion in some cases and may be critical to avoid repetitions and unnecessary testing that may delay diagnosis.

On the contrary, the quantification of circulating dexamethasone levels simultaneously with cortisol levels in the DST to establish whether or not the achieved level of dexamethasone is appropriate for equivocal cases allows avoiding of uncertainties

Table 2. Considerations regarding mass spectrometry measurements for Cushing's syndrome (CS) evaluation

\begin{tabular}{lc}
\hline Advantage provided by mass spectrometry & References \\
\hline Cortisol measurement results are more specific. In contrast to immunoassays, only true cortisol is measured. & {$[6,14,25-29,48,56,60,61,73,85]$} \\
The variability of cortisol measurements across laboratories is reduced. & {$[2,25,45,71,73]$} \\
Clinical value for the screening of CS is high. Comparable (but probably not better) to many immunoassays. & {$[2,26,32,61]$} \\
May be used to reduce false-positive results obtained through immunoassays. & {$[2,26,57]$} \\
Allows the detection of individually exogenous corticosteroids. & {$[3,36-38]$} \\
Ensures adequate plasma dexamethasone levels during DST in equivocal cases. & {$[6,69,70]$} \\
Allows accurate cortisol measurement in patients undergoing interfering medical treatment. & {$[72]$} \\
Allows measurement of endogenous metabolites that may be useful for the following purposes: & {$[3,39,40]$} \\
- subtyping CS & {$[75]$} \\
- classifying adrenal incidentalomes & {$[42]$} \\
- detecting subclinical CS & {$[74]$} \\
- differentiating Cushing's disease from ectopic ACTH syndrome & {$[43]$} \\
- detection of saliva contamination & {$[62,63]$} \\
\hline
\end{tabular}

Abbreviations: ACTH, adrenocorticotropic hormone; CS, Cushing's syndrome; DST, dexamethasone suppression test. 
regarding drug compliance or absorption/metabolic effects on the drug levels. In addition, patients with CS under medical treatment may present large variations in their metabolites and may thus benefit from mass spectrometric measurements during follow-up. Finally, mass spectrometry measurement of selected endogenous metabolites and comprehensive steroid profiling have been proposed to be useful in the detection of subclinical CS and saliva contamination and for the classification of CS and adrenal incidentalomes (Table 2).

Future work areas include the use of mass spectrometry-based methods to achieve an improvement in diagnostic sensitivity, a key requirement for CS screening, for which immunoassays are likely to continue to play a predominant role in the near future. However, as presented here, the use of mass spectrometry for the diagnosis of CS in clinical laboratories clearly allows for the reduction of many diagnostic uncertainties related with this complex disease while allowing a better classification of the patients, which undoubtedly will contribute to a better diagnosis and management of patients with CS.

\section{ACKNOWLEDGEMENTS}

None.

\section{AUTHOR CONTRIBUTIONS}

F.A.H. and G.C prepared and wrote the review manuscript.

\section{CONFLICTS OF INTEREST}

The authors declare no conflict of interest.

\section{RESEARCH FUNDING}

None declared.

\section{ORCID}

Gregori Casals https://orcid.org/0000-0002-3271-1371

Felicia Alexandra Hanzu https://orcid.org/0000-0002-2399-4023

\section{REFERENCES}

1. Lacroix A, Feelders RA, Stratakis CA, Nieman LK. Cushing's syndrome. Lancet 2015;386:913-27.

2. Clayton RN, Raskauskiene D, Reulen RC, Jones PW. Mortality and morbidity in Cushing's disease over 50 years in Stoke-on-Trent, UK: audit and meta-analysis of literature. J Clin Endocrinol Metab 2011;96:63242.

3. Neary NM, Booker OJ, Abel BS, Matta JR, Muldoon N, Sinaii N, et al. Hypercortisolism is associated with increased coronary arterial atherosclerosis: analysis of noninvasive coronary angiography using multidetector computerized tomography. J Clin Endocrinol Metab 2013;98:204552.

4. Loriaux DL. Diagnosis and differential diagnosis of Cushing's syndrome. N Engl J Med 2017;376:1451-9.

5. Pappachan JM, Hariman C, Edavalath M, Waldron J, Hanna FW. Cushing's syndrome: a practical approach to diagnosis and differential diagnoses. J Clin Pathol 2017;70:350-9.

6. Chabre O. The difficulties of pseudo-Cushing's syndrome (or 'non-neoplastic hypercortisolism'). Ann Endocrinol (Paris) 2018;79:138-45.

7. Jung C, Ho JT, Torpy DJ, Rogers A, Doogue M, Lewis JG, et al. A longitudinal study of plasma and urinary cortisol in pregnancy and postpartum. J Clin Endocrinol Metab 2011;96:1533-40.

8. Chiodini I. Clinical review: diagnosis and treatment of subclinical hypercortisolism. J Clin Endocrinol Metab 2011;96:1223-36.

9. Meinardi JR, Wolffenbuttel BH, Dullaart RP. Cyclic Cushing's syndrome: a clinical challenge. Eur J Endocrinol 2007;157:245-54.

10. Manenschijn L, Koper JW, van den Akker EL, de Heide LJ, Geerdink $\mathrm{EA}$, de Jong $\mathrm{FH}$, et al. A novel tool in the diagnosis and follow-up of (cyclic) Cushing's syndrome: measurement of long-term cortisol in scalp hair. J Clin Endocrinol Metab 2012;97:E1836-43.

11. Hodes A, Meyer J, Lodish MB, Stratakis CA, Zilbermint M. Mini-review of hair cortisol concentration for evaluation of Cushing syndrome. Expert Rev Endocrinol Metab 2018;13:225-31.

12. Greff MJE, Levine JM, Abuzgaia AM, Elzagallaai AA, Rieder MJ, van Uum SHM. Hair cortisol analysis: an update on methodological considerations and clinical applications. Clin Biochem 2019;63:1-9.

13. Juszczak A, Sulentic P, Grossman A. Cushing's syndrome. In: Feingold KR, Anawalt B et al., eds. Endotext [Internet]. South Dartmouth (MA): MDText.com, Inc., 2000-2017.

14. Nieman LK, Biller BM, Findling JW, Newell-Price J, Savage MO, Stewart PM et al. The diagnosis of Cushing's syndrome: an Endocrine Society Clinical Practice Guideline. J Clin Endocrinol Metab 2008:93:1526-40.

15. Arnaldi G, Angeli A, Atkinson AB, Bertagna X, Cavagnini F, Chrousos GP, et al. Diagnosis and complications of Cushing's syndrome: a consensus statement. J Clin Endocrinol Metab 2003;88:5593-602.

16. Barbot M, Trementino L, Zilio M, Ceccato F, Albiger N, Daniele A, et al. Second-line tests in the differential diagnosis of ACTH-dependent Cushing's syndrome. Pituitary 2016;19:488-95.

17. Aranda G, Enseñat J, Mora M, Puig-Domingo M, Martínez de Osaba MJ, Casals $\mathrm{G}$, et al. Long-term remission and recurrence rate in a cohort of Cushing's disease: the need for long-term follow-up. Pituitary 2015;18: 142-9.

18. Yeo KT, Babic N, Hannoush ZC, Weiss RE. Endocrine testing protocols: hypothalamic pituitary adrenal axis. In: Feingold KR, Anawalt B, et al., eds. Endotext [Internet]. South Dartmouth, MA: MDText.com, Inc.:20002017.

19. Deutschbein T, Broecker-Preuss M, Hartmann MF, Althoff R, Wudy SA, Mann K, et al. Measurement of urinary free cortisol by current immunoassays: need for sex-dependent reference ranges to define hypercortisolism. Horm Metab Res 2011;43:714-9.

20. Miki $\mathrm{K}$ and Sudo A. Effect of urine $\mathrm{pH}$, storage time, and temperature on stability of catecholamines, cortisol, and creatinine. Clin Chem 1998; 44:1759-62.

21. Hansen AM, Garde AH, Skovgaard LT, Christensen JM. Seasonal and biological variation of urinary epinephrine, norepinephrine, and cortiso 
in healthy women. Clin Chim Acta 2001;309:25-35.

22. Pecori Giraldi F and Ambrogio AG. Variability in laboratory parameters used for management of Cushing's syndrome. Endocrine 2015;50:580-9.

23. Chan KC, Lit LC, Law EL, Tai MH, Yung CU, Chan MH, et al. Diminished urinary free cortisol excretion in patients with moderate and severe renal impairment. Clin Chem 2004;50:757-9.

24. Turpeinen $U$ and Hämäläinen E. Determination of cortisol in serum, saliva and urine. Best Pract Res Clin Endocrinol Metab 2013;27:795-801.

25. Wood L, Ducroq DH, Fraser HL, Gillingwater S, Evans C, Pickett AJ, et al. Measurement of urinary free cortisol by tandem mass spectrometry and comparison with results obtained by gas chromatography-mass spectrometry and two commercial immunoassays. Ann Clin Biochem 2008;45:380-8.

26. Aranda G, Careaga M, Hanzu FA, Patrascioiu I, Ríos P, Mora M, et al. Accuracy of immunoassay and mass spectrometry urinary free cortisol in the diagnosis of Cushing's syndrome. Pituitary 2016;19:496-502.

27. McCann SJ, Gillingwater S, Keevil BG. Measurement of urinary free cortisol using liquid chromatography-tandem mass spectrometry: comparison with the urine adapted ACS:180 serum cortisol chemiluminescent immunoassay and development of a new reference range. Ann Clin Biochem 2005;42:112-8.

28. Ceccato F, Barbot M, Zilio M, Frigo AC, Albiger N, Camozzi V, et al. Screening tests for Cushing's syndrome: urinary free cortisol role measured by LC-MS/MS. J Clin Endocrinol Metab 2015;100:3856-61.

29. Ceccato F, Antonelli G, Barbot M, Zilio M, Mazzai L, Gatti R, et al. The diagnostic performance of urinary free cortisol is better than the cortisol: cortisone ratio in detecting de novo Cushing's syndrome: the use of a LC-MS/MS method in routine clinical practice. Eur J Endocrinol 2014; 171:1-7.

30. Murphy BE. How much "UFC" is really cortisol? Clin Chem 2000;46: 793-4.

31. Raff H, Auchus RJ, Findling JW, Nieman LK. Urine free cortisol in the diagnosis of Cushing's syndrome: is it worth doing and, if so, how? J Clin Endocrinol Metab 2015;100:395-7.

32. OBwald A, Wang R, Beuschlein F, Hartmann MF, Wudy SA, Bidlingmaier M, et al. Performance of LC-MS/MS and immunoassay based 24-h urine free cortisol in the diagnosis of Cushing's syndrome. J Steroid Biochem Mol Biol 2019;190:193-7.

33. El-Farhan N, Rees DA, Evans C. Measuring cortisol in serum, urine and saliva-are our assays good enough? Ann Clin Biochem 2017;54:30822.

34. Roberts RF and Roberts WL. Performance characteristics of five automated serum cortisol immunoassays. Clin Biochem 2004;37:489-93.

35. Fink RS, Pierre LN, Daley-Yates PT, Richards DH, Gibson A, Honour JW. Hypothalamic-pituitary-adrenal axis function after inhaled corticosteroids: unreliability of urinary free cortisol estimation. J Clin Endocrinol Metab 2002;87:4541-6.

36. Thynne T, White GH, Burt MG. Factitious Cushing's syndrome masquerading as Cushing's disease. Clin Endocrinol (Oxf) 2014;80:328-32.

37. Djedovic NK and Rainbow SJ. Detection of synthetic glucocorticoids by liquid chromatography-tandem mass spectrometry in patients being investigated for Cushing's syndrome. Ann Clin Biochem 2011;48:542-9.

38. Taylor RL, Grebe SK, Singh RJ. Quantitative, highly sensitive liquid chromatography-tandem mass spectrometry method for detection of synthetic corticosteroids. Clin Chem 2004;50:2345-52.

39. Krone N, Hughes BA, Lavery GG, Stewart PM, Arlt W, Shackleton CH. Gas chromatography/mass spectrometry (GC/MS) remains a pre-eminent discovery tool in clinical steroid investigations even in the era of fast liquid chromatography tandem mass spectrometry (LC/MS/MS). J Steroid Biochem Mol Biol 2010;121:496-504.
40. Marcos J, Renau N, Casals G, Segura J, Ventura R, Pozo OJ. Investigation of endogenous corticosteroids profiles in human urine based on liquid chromatography tandem mass spectrometry. Anal Chim Acta 2014; 812:92-104.

41. Casals G, Marcos J, Pozo OJ, Alcaraz J, Martínez de Osaba MJ, Jiménez W. Microwave-assisted derivatization: application to steroid profiling by gas chromatography/mass spectrometry. J Chromatogr B Analyt Technol Biomed Life Sci 2014;960:8-13.

42. Brossaud J, Ducint D, Corcuff JB. Urinary glucocorticoid metabolites: biomarkers to classify adrenal incidentalomas? Clin Endocrinol (Oxf) 2016; 84:236-43.

43. Ceccato F, Trementino L, Barbot M, Antonelli G, Plebani M, Denaro L, et al. Diagnostic accuracy of increased urinary cortisol/cortisone ratio to differentiate ACTH-dependent Cushing's syndrome. Clin Endocrinol (Oxf) 2017;87:500-7.

44. Kulle AE, Welzel M, Holterhus PM, Riepe FG. Principles and clinical applications of liquid chromatography-tandem mass spectrometry for the determination of adrenal and gonadal steroid hormones. J Endocrinol Invest 2011;34:702-8.

45. Brossaud J, Leban M, Corcuff JB, Boux de Casson F, Leloupp AG, Masson D, et al. LC-MSMS assays of urinary cortisol, a comparison between four in-house assays. Clin Chem Lab Med 2018;56:1109-16.

46. Carroll T, Raff H, Findling JW. Late-night salivary cortisol for the diagnosis of Cushing syndrome: a meta-analysis. Endocr Pract 2009;15:33542.

47. Elamin MB, Murad MH, Mullan R, Erickson D, Harris K, Nadeem S, et al. Accuracy of diagnostic tests for Cushing's syndrome: a systematic review and metaanalyses. J Clin Endocrinol Metab 2008;93:1553-62.

48. Raff $\mathrm{H}$ and Phillips JM. Bedtime salivary cortisol and cortisone by LCMS/MS in healthy adult subjects: evaluation of sampling time. J Endocr Soc 2019;3:1631-40.

49. Baid SK, Sinaii N, Wade M, Rubino D, Nieman LK. Radioimmunoassay and tandem mass spectrometry measurement of bedtime salivary cortisol levels: a comparison of assays to establish hypercortisolism. J Clin Endocrinol Metab 2007;92:3102-7.

50. Casals G, Foj L, de Osaba MJ. Day-to-day variation of late-night salivary cortisol in healthy voluntaries. Clin Biochem 2011;44:665-8.

51. Sandouk Z, Johnston P, Bunch D, Wang S, Bena J, Hamrahian A, et al. Variability of late-night salivary cortisol in Cushing disease: A prospective study. J Clin Endocrinol Metab 2018;103:983-90.

52. Coelli S, Farias CB, Soares AA, Crescente GM, Hirakata VN, Souza LB, et al. Influence of age, gender and body mass index on late-night salivary cortisol in healthy adults. Clin Chem Lab Med 2017;55:1954-61.

53. Inder WJ, Dimeski G, Russell A. Measurement of salivary cortisol in 2012laboratory techniques and clinical indications. Clin Endocrinol (Oxf) 2012; 77:645-51.

54. Calvi JL, Chen FR, Benson VB, Brindle E, Bristow M, De A, et al. Measurement of cortisol in saliva: a comparison of measurement error within and between international academic-research laboratories. BMC Res Notes 2017;10:479.

55. Deutschbein T, Broecker-Preuss M, Flitsch J, Jaeger A, Althoff R, Walz MK, et al. Salivary cortisol as a diagnostic tool for Cushing's syndrome and adrenal insufficiency: improved screening by an automatic immunoassay. Eur J Endocrinol 2012;166:613-8.

56. Vogeser M, Durner J, Seliger E, Auernhammer C. Measurement of latenight salivary cortisol with an automated immunoassay system. Clin Chem Lab Med 2006;44:1441-5.

57. Ceccato F, Marcelli G, Martino M, Concettoni C, Brugia M, Trementino L, et al. The diagnostic accuracy of increased late night salivary cortisol for Cushing's syndrome: a real-life prospective study. J Endocrinol Invest 


\section{9;42:327-35}

58. Monaghan PJ, Keevil BG, Trainer PJ. The use of mass spectrometry to improve the diagnosis and the management of the HPA axis. Rev Endocr Metab Disord 2013;14:143-57.

59. Beko G, Varga I, Glaz E, Sereg M, Feldman K, Toth M, et al. Cutoff values of midnight salivary cortisol for the diagnosis of overt hypercortisolism are highly influenced by methods. Clin Chim Acta 2010;411:364-7.

60. Raff H. Cushing's syndrome: diagnosis and surveillance using salivary cortisol. Pituitary 2012;15:64-70.

61. Mészáros K, Karvaly G, Márta Z, Magda B, Tőke J, Szücs N, et al. Diagnostic performance of a newly developed salivary cortisol and cortisone measurement using an LC-MS/MS method with simple and rapid sample preparation. J Endocrinol Invest 2018;41:315-23.

62. Kline GA, Buse JD, Van Der Gugten JG, Holmes DT, Chin AC, Sadrzadeh SMH. Factitious ACTH-dependent, apparent hypercortisolism: the problem with late-night salivary cortisol measurements collected at home. Clin Endocrinol (Oxf) 2017;87:882-5.

63. Raff $\mathrm{H}$ and Singh RJ. Measurement of late-night salivary cortisol and cortisone by LC-MS/MS to assess preanalytical sample contamination with topical hydrocortisone. Clin Chem 2012;58:947-8.

64. Israelsson M, Brattsand R, Brattsand G. 20 $\alpha$ - and 20 $\beta$-dihydrocortisone may interfere in LC-MS/MS determination of cortisol in saliva and urine. Ann Clin Biochem 2018;55:341-7.

65. Fassnacht M, Arlt W, Bancos I, Dralle H, Newell-Price J, Sahdev A, et al. Management of adrenal incidentalomas: European Society of Endocrinology Clinical Practice Guideline in collaboration with the European Network for the Study of Adrenal Tumors. Eur J Endocrinol 2016;175: G1-34.

66. Nieman LK. Recent updates on the diagnosis and management of Cushing's syndrome. Endocrinol Metab (Seoul) 2018;33:139-46.

67. Vastbinder M, Kuindersma M, Mulder AH, Schuijt MP, Mudde AH. The influence of oral contraceptives on overnight $1 \mathrm{mg}$ dexamethasone suppression test. Neth J Med 2016;74:158-61.

68. Meikle AW. Dexamethasone suppression tests: usefulness of simultaneous measurement of plasma cortisol and dexamethasone. Clin Endocrinol (Oxf) 1982;16:401-8.

69. Hempen C, Elfering S, Mulder AH, van den Bergh FA, Maatman RG. Dexamethasone suppression test: development of a method for simultaneous determination of cortisol and dexamethasone in human plasma by liquid chromatography/tandem mass spectrometry. Ann Clin Biochem 2012;49:170-6.

70. Ueland GÅ, Methlie P, Kellmann R, Bjørgaas M, Åsvold BO, Thorstensen $\mathrm{K}$, et al. Simultaneous assay of cortisol and dexamethasone improved diagnostic accuracy of the dexamethasone suppression test. Eur J Endocrinol 2017;176:705-13.

71. Brixey-McCann R, Tennant SM, Geen J, Armston A, Barth JH, Keevil B, et al. Effect of cortisol assay bias on the overnight dexamethasone suppression test: implications for the investigation of Cushing's syndrome. Endocrine Abstracts 2015;38:22.

72. Monaghan PJ, Owen LJ, Trainer PJ, Brabant G, Keevil BG, Darby D. Comparison of serum cortisol measurement by immunoassay and liquid chromatography-tandem mass spectrometry in patients receiving the $11 \beta$ hydroxylase inhibitor metyrapone. Ann Clin Biochem 2011;48:441-6.

73. El-Farhan N, Pickett A, Ducroq D, Bailey C, Mitchem K, Morgan N, et al. Method-specific serum cortisol responses to the adrenocorticotrophin test: comparison of gas chromatography-mass spectrometry and five automated immunoassays. Clin Endocrinol (Oxf) 2013;78:673-80.

74. Masjkur J, Gruber M, Peitzsch M, Kaden D, Di Dalmazi G, Bidlingmaier $M$, et al. Plasma steroid profiles in subclinical compared with overt adrenal Cushing syndrome. J Clin Endocrinol Metab 2019;104:4331-40.

75. Eisenhofer G, Masjkur J, Peitzsch M, Di Dalmazi G, Bidlingmaier M, Grüber $\mathrm{M}$, et al. Plasma steroid metabolome profiling for diagnosis and subtyping patients with Cushing syndrome. Clin Chem 2018;64:586-96.

76. Raff H, Raff JL, Findling JW. Late-night salivary cortisol as a screening test for Cushing's syndrome. J Clin Endocrinol Metab 1998;83:2681-6.

77. Papanicolaou DA, Mullen N, Kyrou I, Nieman LK. Nighttime salivary cortisol: a useful test for the diagnosis of Cushing's syndrome. J Clin Endocrinol Metab 2002;87:4515-21.

78. Putignano P, Toja P, Dubini A, Pecori Giraldi F, Corsello SM, Cavagnini F. Midnight salivary cortisol versus urinary free and midnight serum cortisol as screening tests for Cushing's syndrome. J Clin Endocrinol Metab 2003;88:4153-7.

79. Yaneva M, Mosnier-Pudar H, Dugué MA, Grabar S, Fulla Y, Bertagna X. Midnight salivary cortisol for the initial diagnosis of Cushing's syndrome of various causes. J Clin Endocrinol Metab 2004;89:3345-51.

80. Viardot A, Huber P, Puder JJ, Zulewski H, Keller U, Müller B. Reproducibility of nighttime salivary cortisol and its use in the diagnosis of hypercortisolism compared with urinary free cortisol and overnight dexamethasone suppression test. J Clin Endocrinol Metab 2005;90:5730-6.

81. Bukan AP, Dere HB, Jadhav SS, Kasaliwal RR, Budyal SR, Shivane VK, et al. The performance and reproducibility of late-night salivary cortisol estimation by enzyme immunoassay for screening Cushing disease. Endocr Pract 2015;21:158-64.

82. Findling JW, Fleseriu M, Newell-Price J, Petersenn S, Pivonello R, Kandra A, et al. Late-night salivary cortisol may be valuable for assessing treatment response in patients with Cushing's disease: 12-month, Phase III pasireotide study. Endocrine 2016;54:516-23.

83. Ambroziak U, Kondracka A, Bartoszewicz Z, Krasnodębska-Kiljańska M, Bednarczuk T. The morning and late-night salivary cortisol ranges for healthy women may be used in pregnancy. Clin Endocrinol (Oxf) 2015; 83:774-8.

84. Lages AS, Frade JG, Oliveira D, Paiva I, Oliveira P, Rebelo-Marques A, et al. Late-night salivary cortisol: cut-off definition and diagnostic accuracy for Cushing's syndrome in a Portuguese population. Acta Med Port 2019;32:381-7.

85. Erickson D, Singh RJ, Sathananthan A, Vella A, Bryant SC. Late-night salivary cortisol for diagnosis of Cushing's syndrome by liquid chromatography/tandem mass spectrometry assay. Clin Endocrinol (Oxf) 2012; 76:467-72.

86. Palmieri S, Morelli V, Polledri E, Fustinoni S, Mercadante R, Olgiati L, et al. The role of salivary cortisol measured by liquid chromatography-tandem mass spectrometry in the diagnosis of subclinical hypercortisolism. Eur J Endocrinol 2013;168:289-96.

87. Antonelli G, Ceccato F, Artusi C, Marinova M, Plebani M. Salivary cortisol and cortisone by LC-MS/MS: validation, reference intervals and diagnostic accuracy in Cushing's syndrome. Clin Chim Acta 2015;451: 247-51. 